\title{
Spontaneous splenic rupture in typhoid fever
}

\author{
Gazanfar Ali, M.A. Kamili, Samia Rashid, Aijaz Mansoor, Bashir A. Lone \\ and G.Q. Allaqaband
}

Government Medical College, SMHS Hospital, Srinagar-Kashmir, India

\begin{abstract}
Summary: Three cases of multidrug-resistant Salmonella typhi infection presenting as spontaneous splenic rupture are presented. One patient died and two recovered completely. This is a previously unreported presentation of typhoid fever.
\end{abstract}

\section{Introduction}

Spontaneous splenic rupture is a rare complication of various infections, ${ }^{1}$ though typhoid fever can rarely cause splenic rupture. ${ }^{2}$ Typhoid fever presenting as spontaneous splenic rupture has not been previously reported to our knowledge. We report three cases of multidrug-resistant Salmonella typhi infection presenting as spontaneous splenic rupture.

\section{Case reports}

\section{Case 1}

An 18 year old unmarried girl was admitted to the hospital with a history of 2 days' fever and left upper abdominal pain. On examination the patient was febrile, toxic and sweating; pulse rate was $120 /$ minute, blood pressure $110 / 70 \mathrm{mmHg}$. She had a guarded tender abdomen and spleen palpable $2 \mathrm{~cm}$ below the costal margin. The rest of the examination was normal. Leucocyte count was 4.6 with $52 \%$ lymphocytes. Platelet count, urine analysis, and X-rays of chest and abdomen were normal. Blood was sent for culture. Within 2 hours of hospitalization she developed pallor, sweating, altered level of consciousness, shock and abdominal distension. Abdominocentesis revealed bloody fluid with no organism seen on Gram stain. Resuscitative measures were instituted and laparotomy performed. The peritoneal cavity was filled with blood. The spleen was enlarged, friable and had ruptured. Blood culture yielded Salmonella typhi sensitive only to quinolones. The patient developed cardiac arrest and died.

Correspondence: Gazanfar Ali, M.D., T-14 Tulsi Bagh, Srinagar-Kashmir 190 008, India.

Accepted: 25 November 1993

\section{Case 2}

A 45 year old man was admitted to the hospital with fever of 3 weeks and pain in the abdomen of one day's duration. On admission he was toxic, febrile and pale with a fast feeble pulse and blood pressure of $70 / 50 \mathrm{mmHg}$. He had a tender splenomegaly $6 \mathrm{~cm}$ below the costal margin with a guarded tender abdomen and shifting dullness on percussion. There was no history of trauma. Abdominocentesis revealed blood. Resuscitation with intravenous fluids, fresh blood and dopamine stabilized the patient. Laparotomy showed a large friable spleen which had ruptured. The peritoneal cavity was full of blood. Splenectomy was performed and the patient was given ciprofloxacin. Blood culture yielded Salmonella typhi sensitive to ciprofloxacin. The postoperative period was uneventful.

\section{Case 3}

A 52 year old man with a 6 day history of fever presented with severe abdominal pain and vomiting of 3 hours duration. On physical examination he was febrile, pale, sweating, drowsy with a heart rate of $110 /$ minute and blood pressure of $80 / 60 \mathrm{mmHg}$. The abdomen was guarded and tender. The spleen was palpable $5 \mathrm{~cm}$ below the costal margin. His rectal examination was normal. His investigations revealed a haematocrit of $25 \%$, leucocyte count 8.4 with $49 \%$ lymphocytes, and platelet count 1.5. Ultrasonography showed free fluid and splenomegaly. Abdominocentesis yielded blood only. On exploratory laparotomy the peritoneal cavity was filled with blood. The spleen was enlarged, friable and ruptured with active bleeding. Splenectomy was performed and blood evacuated from the peritoneal cavity. Blood culture was positive for Salmonella typhi sensitive to 
ciprofloxacin. He gradually recovered with ciprofloxacin.

\section{Discussion}

Spontaneous splenic rupture is known to occur in infections, inflammatory conditions, haematological disorders, malignancies and collagen vascular diseases. ${ }^{3}$ Various infections causing spontaneous splenic rupture include typhoid, ${ }^{2}$ mumps, ${ }^{3}$ infectious mononucleosis, ${ }^{4}$ infective endocarditis, ${ }^{5}$ malaria,${ }^{6}$ hepatitis, ${ }^{7}$ influenza, ${ }^{8}$ chicken pox ${ }^{9}$ and aspergillosis. ${ }^{10}$ These three cases presented as splenic rupture, a presentation of typhoid fever that has not been previously reported to our

\section{References}

1. Griffith, R.C. \& Janney, C.G. Haematopoietic system: bone marrow and blood, spleen and lymph nodes. In: Kissane, J.M. (ed.) Anderson's Pathology, 9th edn., Vol. 2. The C.V. Mosby Company, St Louis, 1990, pp. 1373-1492.

2. Haynes, B.F. Enlargement of lymph nodes and spleen. In: Wilson, J.D. et al. (eds) Harrison's Principles of Internal Medicine, 12th edn., Vol. 1. McGraw Hill, New York, 1991, pp. 353-359.

3. Massad, M., Murr, M., Razzouk, B., Nassourah, Z., Sankari, M. \& Najjar, F. Spontaneous splenic rupture in an adult with mumps: a case report. Surg 1988, 103: 381.

4. Gillet, M., Pages, C. \& Barbier, E. Splenic rupture and infectious mononucleosis. Med Chir Dig 1974, 3: 105-107.

5. Vergne, R., Selland, B. \& Gobel, F.L. Rupture of spleen in infective endocarditis. Arch Intern Med 1975, 135: 1265-1267.

6. Schwartz, S. (ed.). Principles of Surgery, 3rd edn. New York, McGraw Hill, 1979, p. 1386.

7. Van-Landingham, S.B., Rawls, D.E. \& Roberts, J.W. Pathological rupture of spleen associated with hepatitis A. Arch Surg 1984, 119: 224-225.

8. Vizer, G. \& Weiland, O. Spontaneous splenic rupture in influenza. Orv Hetil 1979, 120: 1139-1140. knowledge. Subcapsular haematomas can occur rarely in typhoid fever as also in infectious mononucleosis and malaria. ${ }^{2}$ The spleen becomes occasionally so soft and swollen in typhoid fever that it can rupture spontaneously. ${ }^{1}$

During the last few decades a number of studies have demonstrated newer facets of typhoid fever, and in the subcontinent attention is being drawn to various known and previous unknown complications. ${ }^{11}$ Because of the emergence of multidrugresistant strains, ${ }^{12,13}$ a more serious profile of typhoid is emerging. ${ }^{14,15}$ The present cases emphasize the need to consider typhoid fever in patients presenting with fever and left upper quadrant abdominal pain, tenderness and/or shock.

9. Lal, P. \& Popple, L.E. Spontaneous rupture of chicken pox spleen. A case report. Br J Surg 1984, 71: 351.

10. Vernant, J.P., Thibault, M., Cordonnier, C. et al. Splenic rupture due to aspergillosis. Nouv Rev Fr Hematol 1983, 25: 385-386.

11. Khosla, S.N. Typhoid fevers - the changing trends. ICMR Bull 1992, 22: 57-65.

12. Kamili, M.A., Gazanfer, A., Shah, M.Y., Samia, R., Khan, S. \& Allaqaband, G.Q. Multiple drug resistant typhoid fever outbreak in Kashmir valley. Indian J Med Sci 1993, 47: $147-151$.

13. Sengupta, S.R. Emergence of drug resistance in salmonellosis Editorial. JAPI 1991, 39: 439-440.

14. Khosla, S.N. Severe typhoid fever with high mortality: is the clinical disease worsening. In: Louf, E.J. (ed.) Proceedings of the XI International Congress for Tropical Medicine and Malaria. University of Calgary, Calgary, Alberta, 1984, p. 75.

15. Gazanfar, A., Kamili, M.A., Shah, M.Y. et al. Neuropsychiatric manifestations of typhoid fever. JAPI 1992, 40: 333-335. 